\title{
A study of medical problems and their outcome in the elderly patients attending the geriatric out patient department of a tertiary care teaching hospital in south India
}

\author{
D Ravi Sankar'1, D. S. Sujith Kumar,"* \\ ${ }^{\mathbf{1}}$ Assistant Professor, ${ }^{\mathbf{2}}$ Associate Professor, Dept. of Community Medicine, SVIMS, Sri Padmavathi Medical College for Women, Tirupati, \\ Andhra Pradesh, India
}

*Corresponding Author: D. S. Sujith Kumar

Email: doctorsujith@gmail.com

\begin{abstract}
Introduction: The health needs and medical needs of elderly populations are different from the young. The current study is intended to study the medical problems with which the elderly patients are attending the outpatient department of a tertiary care teaching hospital and their outcomes.

Materials and Methods: A retrospective record based study was conducted among patients attending the geriatric OPD of tertiary care teaching hospital for a period of six months. Details regarding Medical condition, hospitalization status and treatment outcome were collected. Result: 60-64 years age group patients are the majority followed by 65-69 and 70-75 years age groups. Hypertension is the most common medical condition followed by Diabetes and Musculoskeletal disorders Hypertension being the major disorder in both the genders. 80 (13\%) of them were admitted out of which $5(6.25 \%)$ died during the course of treatment and $75(93.75 \%)$ were treated successfully and discharged. Conclusion: Non-communicable diseases are the predominant geriatric health problems. There is significant gender differences in hospital admission rates but not in hospital death rates. The policy makers can utilize the data from studies like this to take necessary steps for the prevention, control and management of age related chronic diseases by various medical and non-medical measures. Provision of compulsory health insurance for all the elderly and provision of geriatric clinics in all levels of health care will decrease elderly morbidity and mortality.
\end{abstract}

Keywords: Elderly patients, Medical problems, Geriatric OPD.

\section{Introduction}

The UN agreed cutoff is $60+$ years to refer to the older or elderly persons. Within the elderly population, further classification like oldest old (normally those 80+) and centenarian $(100+)$ and even super-centenarian $(110+)$ are also made. Over the long run, if fertility continues to decline, the share of the population of working age also declines and that of older persons increases, leading to rising dependency ratios and when this happen, the phenomenon is called the 'demographic burden'. This is an inevitable consequence of demographic transition and every country has to face this problem with development and successful demographic transition. ${ }^{1}$

The number of older persons in the world is projected to be 1.4 billion in 2030 and 2.1 billion in 2050, and could rise to 3.1 billion in $2100 .^{2}$ Total elderly population which was 142 millions in SEAR in 2010, is likely to have a quantum jump of 100 million in next 15 years to cross 242 million mark by $2025 .{ }^{1}$ Over the next few decades, a further increase in the population of older persons is almost inevitable, given the size of the cohorts born in recent decades. Population ageing is projected to have a profound effect on societies, underscoring the fiscal and political pressures that the health care, old-age pension and social protection systems of many countries are likely to face in the coming decades. ${ }^{2}$

Projection studies indicate that the number of $60+$ in India will increase to 100 million in 2013 and to 198 million in 2030. According to 2001 census, there were 75.93 million Indians above the age of sixty years. The projections for next five censuses till the year 2051 are: 96.30 million (2011), 133.32 million (2021), 178.59 (2031), 236.01 million (2041) and 300.96 million (2051). ${ }^{3}$
The health needs and medical needs of elderly populations are different from the young. Many studies have shown that non-communicable diseases are the major cause of morbidity and mortality among elderly in the world. The hospitals should be equipped with the facilities and resources to manage the elderly. A study into the medical problems faced by the elderly attending the outpatient departments will give an idea of the types of problems faced and resources required to manage these problems. The current study is intended to study the medical problems with which the elderly patients are attending the outpatient department of a tertiary care teaching hospital and their outcomes.

\section{Objectives}

1. To study the medical problems of the elderly patients attending the geriatric Outpatient Department

2. To study the outcome of the elderly patients attending the geriatric Outpatient Department

\section{Materials and Methods}

The study was conducted in a tertiary care teaching hospital. The hospital has recently started a geriatric Out Patient Department (OPD) to cater to the elderly patients. The subjects of this study are patients attending the geriatric OPD of this hospital. The duration of the study was for a period of six months from January to June. The study was a retrospective study. Data regarding the patients attending the geriatric OPD of a tertiary care teaching hospital for a period of six months was collected. Their personal details like age and sex were recorded. These patients records were followed up until they left the hospital. Details about the patient 
regarding their Medical condition, hospitalization status and treatment outcome details were collected.

The medical conditions of the patients were grouped as per the organ system involvement into ten groups. Nonspecific medical conditions not falling under any organ system were grouped as "Others". Medical conditions of gastrointestinal system such as gastro-esophageal reflux disease (GERD), gastritis, gastric ulcer, duodenal ulcer, esophageal ulcer, Zollinger Ellison Syndrome (ZES) and Meckel's diverticular ulcer were collectively grouped as "Acid peptic disease (APD)". ${ }^{4}$

The diseases under the International Statistical Classification of Diseases and Related Health Problems 10th Revision (ICD 10) code 150.0. Congestive Heart Failure including Congestive heart disease and Right ventricular failure (secondary to left heart failure) were grouped under the category of "Congestive Heart Failure (CHF)". Diseases coming under ICD 10 code N18. Chronic kidney disease, which includes chronic kidney disease stage 1 to stage 5 , and chronic kidney disease unspecified were included under the group "Chronic kidney disease (CKD)". Diabetes Mellitus in ICD 10 ranging from E10-E14 are included under the "Diabetes Mellitus (DM)" group. Hypertensive patients as per ICD 10 code I10 Essential (primary) hypertension which includes High blood pressure, Hypertension (arterial) (benign) (essential) (malignant) (primary) (systemic) and Excludes involvement of vessels of brain (I60-I69) and eye (H35.0) are grouped as "Hypertensive (HTN)". Patients having both Diabetes Mellitus and Hypertension were grouped under "Diabetes Mellitus and Hypertensive (DM \& HTN)" group. ${ }^{5}$

All the soft-tissue injuries caused by sudden or sustained exposure to repetitive motion, force, vibration, and awkward positions are included in "Musculoskeletal Disorders (MSD)" group as per Musculoskeletal Health Program of The National Institute for Occupational Safety and Health (NIOSH), Centers for Disease Control and Prevention (CDC), Atlanta, UAS. These musculoskeletal disorders can affect the muscles, nerves, tendons, joints and cartilage in your upper and lower limbs, neck and lower back. ${ }^{6}$ Respiratory conditions including acute respiratory infections as well as chronic respiratory diseases, such as asthma, chronic obstructive pulmonary disease and lung cancer were grouped under "Respiratory tract diseases (RTD)" as per Respiratory tract diseases definition of WHO regional office for the east Mediterranean. ${ }^{7}$

Cerebrovascular accidents occurring as Central nervous system infarction or intracerebral hemorrhage or subarachnoid hemorrhage or cerebral venous thrombosis and not otherwise specified Cerebrovascular accidents were grouped under "Stroke" as per An Updated Definition of Stroke for the 21st Century: A Statement for Healthcare Professionals from the American Heart Association/ American Stroke Association. ${ }^{8}$

The data collected was entered into Microsoft excel 2016 MSO (16.0.4738.1000) 64-bit data sheet and analyzed using Epi Info ${ }^{\mathrm{TM}}$ for Windows, Version 7.2, Build 7.2.2.6_Release Date: 02/02/2018. The patients were grouped under different age groups and sexes. Medical conditions in different age groups and sexes were studied in frequencies and percentages. Chi-square goodness of fit test was used to identify any significant differences in medical conditions of patients among different age groups and sexes. The hospitalization status and treatment outcome in the form of death or discharge after treatment was compared among different sexes.

\section{Results and Discussion}

A total number of 615 patients attended the outpatient department. The age of patients ranged from 60 to 90 years with mean and median age of 65 years. Of these, $346(56.2 \%)$ were males and $269(43.7 \%)$ were females. The differences in the sexes among total number of patients is statistically highly significant $(\mathrm{p}=0.0022)$. 60-64 years age group patients are the majority followed by $65-69$ and 70-75 years age groups. Except the 65-69 years age group, males are high in number compared to females. The sex difference is statistically highly significant $(\mathrm{p}<0.0001)$ in $70-79$ age groups but not in other age groups. Hypertension is the most common medical condition followed by Diabetes and Musculoskeletal disorders. Diabetes is the predominant medical condition in the 60-64 years age group while Hypertension is the predominant medical condition in $65+$ age group.

In both the sexes, Hypertension is the most common Medical condition. Hypertension is followed by Diabetes in females while musculoskeletal disorders followed Hypertension as the major medical condition in males. Diabetic and Hypertensive females are more compared to males but the difference is not statistically significant $(p<0.05)$. Except for Diabetes and hypertension, males are having other medical conditions predominantly. The differences in sexes is statistically significant in congestive cardiac failure and respiratory tract diseases but not in other disorders.

Of all the elderly attending the Geriatric OPD, 80 (13\%) of them were admitted. There is statistically highly significant $(p<0.0001)$ difference between OPD and inpatient treatment. Among in-patients $50(62.5 \%)$ were males and $30(37.5 \%)$ were females. The differences in the admission rates among males and females is also statistically significant ( $\mathrm{p}=0.0335)$. Among those admitted as in-patients, $5(6.25 \%)$ died during the course of treatment and 75 $(93.75 \%)$ were treated successfully and discharged. There is statistically highly significant $(\mathrm{p}<0.0001)$ difference between deaths and successfully treated in-patients. Out of the inpatient deaths $4(80 \%)$ were males and one was female. The differences in the death rates among males and females is not statistically significant $(\mathrm{p}=0.3711)$.

The age of patients in this study ranged from 60 to 90 years with mean and median age of 65 years. Of these $45.5 \%$ and $25.7 \%$ were between the 60-64 years and 65-69 years respectively. $56.2 \%$ of the patients were males and $43.7 \%$ were females. The medical problems among the patients were Diabetes (45.5\%), Hypertension (25.7\%), RTD (7.5\%), MSD (14.3\%). In a similar study conducted at Geriatric OPD at 
Rural Health Training centre in Pondicherry showed the median age group of patients attending the geriatric OPD was 65 years. Out of which $54 \%$ and $23 \%$ were between the age group of 60-65 years and 66-70 years respectively. $48.2 \%$ of the patients were males while $51.8 \%$ were females. The chronic morbidities among the patients were Joint pains (60.5\%), Hypertension (20.9\%), Diabetes Mellitus (17.0\%) and Lung disease $(8.8 \%){ }^{9}$

In a cross-sectional study on morbidity pattern among the elderly population in the rural area of Pondicherry showed that majority $(59.2 \%)$ of the elderly were in the age group of $60-69$ followed by $33.6 \%$ in $70-79$ and $7.2 \%$ in 80 and above. The major problem was hypertension (28\%) followed by arthritis (25\%), diabetes (22\%), respiratory diseases including asthma and tuberculosis (9\%), acid peptic diseases $(5 \%)$. In this study majority $(71.2 \%)$ of the elderly were in the age group of $60-69$ years followed by $24.7 \%$ in $70-79$ years and $4.1 \%$ in 80 years and above. In this study the major problem was Diabetes (45.5\%) followed by Hypertension (25.7\%), MSD (14.3\%), RTD (7.5\%) and APD (5.7\%). ${ }^{10}$

A community based health care study for the elderly at Pondicherry found that $28 \%$ of the elderly had hypertension very near to the results of this study. ${ }^{11}$ In a study on Prevalence of health, related disability among community dwelling urban elderly from middle socioeconomic strata in Bengaluru, India showed the distribution of medical conditions as Hypertension (49.4\%), Diabetes (32.3\%), Arthritis (28.1\%), Respiratory diseases (15.4\%) and Stroke $(5.6 \%)$. The study results were similar in case of stroke but not for other medical conditions. ${ }^{12}$

In a study on morbidity pattern among the elderly population in the rural area of Tamil Nadu. India, the largest age group was 60-64 followed by $65-69$ and 70-75 very similar to this study. Percentages of Male and female is approximately inverse to the findings in this study. Complaints of joint pains/joint stiffness in $43.4 \%$, Hypertension in $25.9 \%$ and diabetes in $8.3 \%$, heart illnesses in $9 \%$ and respiratory system in $7.3 \%$. The respiratory disorders in this study match with the above-mentioned study while other disorders do not match. ${ }^{13}$

In a community-based study on the health status of the elderly most of the elderly belonged to 60-70 year age group similar to this study. Only $7 \%$ of the elderly are above 80 years of age, which is very near to this study finding. The mean age was found to be $68 \pm 2 \times 7.5$ (SD), which includes the mean age of this study. Females were $45 \%$ and males were $55 \%$ very near to this study finding. The hypertensive prevalence was found to be at $30.8 \%$ similar to this study. The females contributed to $60 \%$ of Hypertensive cases which is contradictory to this study. ${ }^{14}$

A WHO technical report on Epidemiology and prevention of cardiovascular diseases in elderly people reported that over half of the elderly above 65 years are having hypertension. The values are very high in comparison to this study. The same report goes on to say that people with sustained hypertension could be between one fourths to one thirds, which is similar to this study. ${ }^{15}$

In a study of Medical Problems Referred to a Care of the Elderly Physician, The age of the patients in this study ranged from 54 to 92 , with a mean age of 70.3 years. Almost threequarters of the patients seen were female. Medical problems identified were Hypertension in $32.7 \%$, Cardiovascular diseases in $10.6 \%$, Stroke or transient ischemic attacks in $1.9 \%$, Musculoskeletal and joint pains in 39.4\%, Diabetes in 22.1\%, Asthma / Chronic Obstructive Pulmonary Disease (COPD) (dyspnoea NYD) in 3.9\%. The findings were similar in terms of Hypertension, near to Diabetes, distant in cardiovascular diseases, Stroke and RTD and highly distant in MSDs. ${ }^{16}$

A study on the Pattern and Outcome of medical admissions of elderly patients seen at a University Teaching Hospital showed that $70 \%$ of the elderly admissions were males and 30\% were females. The results are comparable with this study as male admissions were $62.5 \%$ and female admissions were $37.5 \%$. In-hospital mortality was $31.3 \%$, which is approximately five times more than this study. Of these in-hospital deaths $77.27 \%$ were that of men which is close to the findings of this study. ${ }^{17}$

Similar study on the pattern of geriatric admissions in the medical wards at a National Hospital found that the male to female admissions were 1.7:1 very similar to our study. The in-hospital mortality was also very similar to this study at $6.2 \% .{ }^{18}$ In comparison to a study on Gender differences in the utilization of health-care services among the older adult population. While no significant gender differences were observed in the percentage of subjects that were admitted to hospitals, this study found highly significant difference in the genders. 19

Table 1: Distribution of patients in different age groups and sexes $(n=165)$

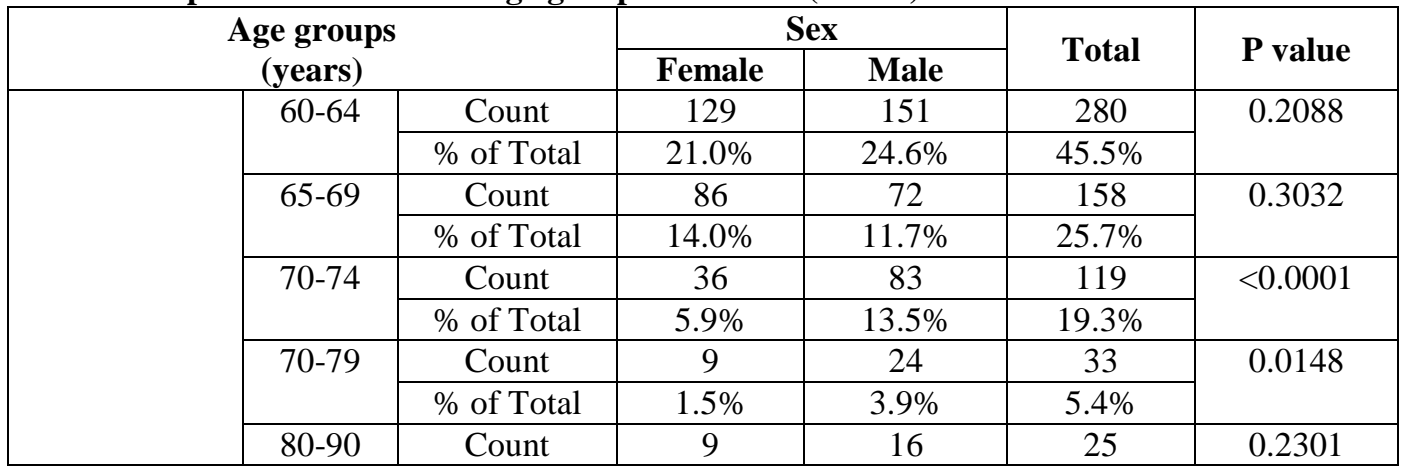




\begin{tabular}{|l|c|r|r|r|r|}
\hline & \% of Total & $1.5 \%$ & $2.6 \%$ & $4.1 \%$ & \\
\hline \multirow{2}{*}{ Total } & Count & 269 & 346 & 615 & \multirow{2}{*}{0.0022} \\
\cline { 2 - 5 } & \% of Total & $43.7 \%$ & $56.3 \%$ & $100.0 \%$ & \\
\hline
\end{tabular}

Table 2: Patients grouped into different medical conditions and sexes $(n=615)$

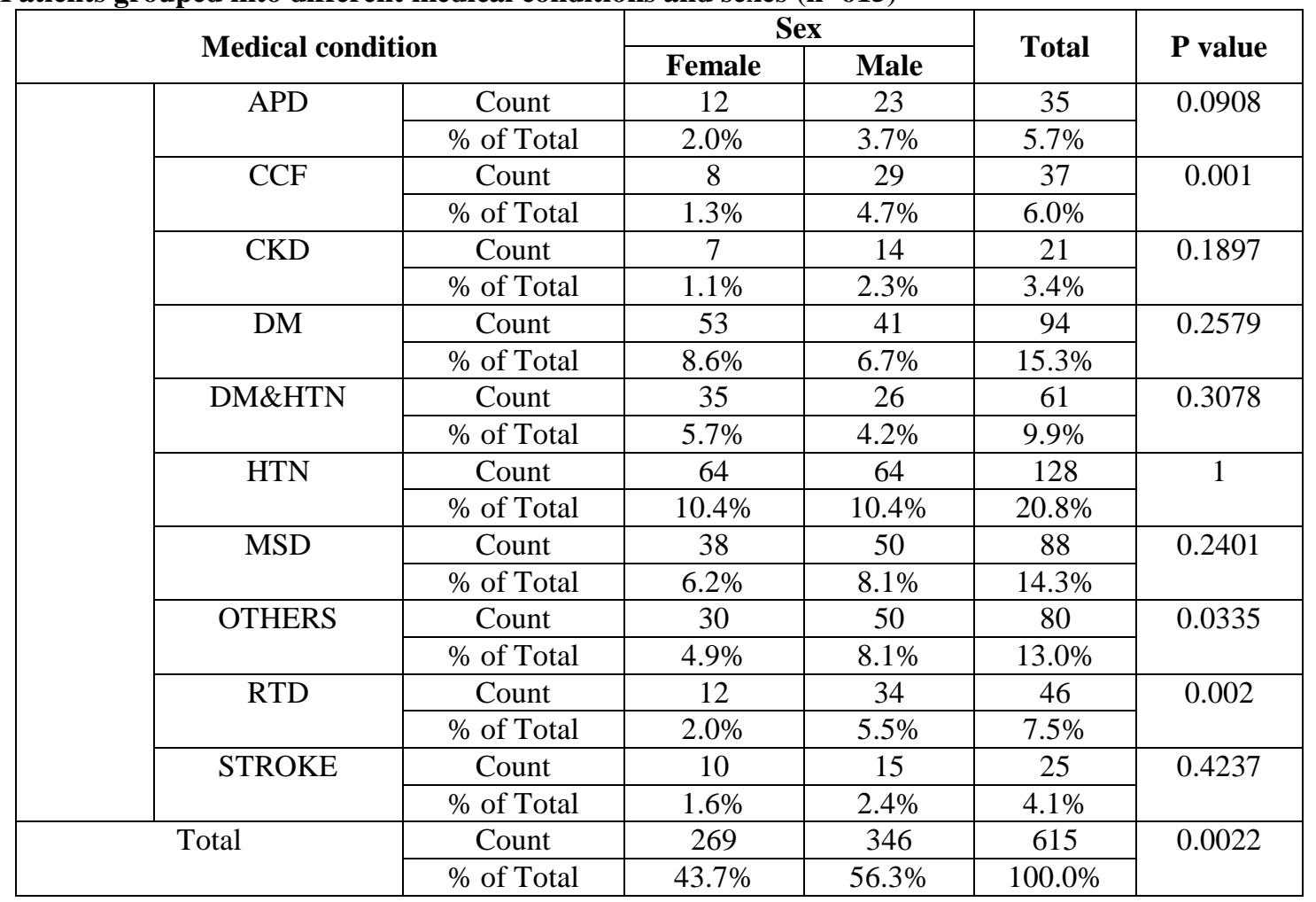

Table 3: Patients outcomes and gender $(n=615)$

\begin{tabular}{|c|c|c|c|c|}
\hline \multicolumn{2}{|c|}{} & Males & Females & Total \\
\hline \multicolumn{2}{|c|}{ Outpatients } & $296(48.13 \%)$ & $239(38.86 \%)$ & $535(87 \%)$ \\
\hline \multirow{2}{*}{ In patients } & Discharged & $46(7.48 \%)$ & $29(4.72 \%)$ & $75(12.2 \%)$ \\
\cline { 2 - 5 } & Died & $4(0.65 \%)$ & $1(0.16 \%)$ & $5(0.8 \%)$ \\
\hline \multicolumn{2}{|c|}{ Total } & $346(56.4 \%)$ & $269(43.7 \%)$ & $615(100 \%)$ \\
\hline
\end{tabular}

\section{Conclusion}

This study concludes that 60-64 years age group patients are the majority followed by 65-69 and 70-75 years age groups attending the geriatric OPD. The gender differences among total number of patients is highly significant. Hypertension is the most common medical condition followed by Diabetes and Musculoskeletal disorders. In both genders, Hypertension is the most common Medical condition. There is highly significant difference between OPD and in-patiently treated. The differences in the admission rates among males and females is also significant. There is highly significant difference between deaths and successfully treated in-patients. The differences in the death rates among in-hospital treated males and females is not significant. The government and policy makers can utilize the data from studies like this to take necessary steps for the prevention, control and management of age related chronic diseases by various medical and non-medical measures.
Provision of compulsory health insurance for all the elderly and provision of geriatric clinics in all levels of health care will decrease elderly morbidity and mortality.

\section{Conflict of Interest: Nil.}

\section{References}

1. Health situation and trend assessment. Elderly population. Trends in proportion of older persons $(60+)$. Available from: http://www.searo.who.int/entity/health_situation_trends/data/c hi/elderly-population/en.

2. United Nations, Department of Economic and Social Affairs, Population Division (2017). World Population Prospects: The 2017 Revision, Volume II: Demographic Profiles (ST/ESA/SER.A/400).

3. Ministry of health and family welfare. New Delhi: Director General of Health Services, MOHFW, Government of India; 2011. National Program for Health Care of the Elderly (NPHCE): Operational Guidelines 2011.

4. KUNMAM (ACID PEPTIC DISEASE). Available from: https://www.nhp.gov.in/Kunmam-(Acid-Peptic-Disease)_mtl. 
5. WHO. International Statistical Classification of Diseases and Related Health Problems 10th Revision. ICD-10 Version:2010. Available from:

http://apps.who.int/classifications/icd10/browse/2010/en.

6. Musculoskeletal Health Program of The National Institute for Occupational Safety and Health (NIOSH), Centers for Disease Control and Prevention (CDC), Atlanta. Available from: https://www.cdc.gov/niosh/programs/msd.

7. Respiratory tract diseases. Available from: http://www.emro.who.int/health-topics/respiratory-tractdiseases/index.html.

8. Ralph L. Sacco et al. An Updated Definition of Stroke for the 21st Century: A Statement for Healthcare Professionals from the American Heart Association/American Stroke Association. Stroke. 2013;44(7):2064-2089.

9. Muruganandham R, Ingole A, Murugan V, Dongre AR. An Approach of Initiating Geriatric Screening OPD at the Rural Health Training Centre of SMVMCH, Pondicherry. Online $J$ Health Allied Scs. 2013;12(4):2.

10. Sudarshan BP, Chethan TK. Morbidity pattern among the elderly population in the rural area of Pondicherry: a cross sectional study. Int J Community Med Public Health. 2016;3:414-418

11. Subramanyam DKS, Premarajan KC, Dutta TK, Soudarssanane BM. Community based health care for the elderly. Project Report Implemented Under The Government of India World Health Organization Collaborative Programme: 2006-2007.

12. Krishnamachari Srinivasan, Mario Vaz, Tinku Thomas. Prevalence of health related disability among community dwelling urban elderly from middle socioeconomic strata in Bangaluru, India. Indian J Med Res. 2010;131:515-521.
13. Purty AJ, Bazroy J, Kar M, Vasudevan K, Veliath A, Panda P. Morbidity pattern among the elderly population in the rural area of Tamil Nadu. India. Turk J Med Sci. 2006;36:45-50.

14. Saxena $\mathrm{V}$ et al. health status of the elderly- A community Based study. Indian J Community Health. 2012;24(4):269-274.

15. WHO. Epidemiology and prevention of cardiovascular diseases in elderly people. Report of a WHO study group. Technical report series 853, Geneva 1995.

16. Lam R, Gallinaro A, Adleman J. Medical Problems Referred to a Care of the Elderly Physician: Insight for Future Geriatrics CME. Can Geriatr J. 2013;16(3):114-119.

17. EO Sanya, TM Akande, G Opadijo, JK Olarinoye, BJ Bojuwoye. Pattern and outcome of medical admission of elderly patients seen at University of Ilorin Teaching Hospital, Ilorin. Afr J Med Med Sci. 2008;37:375-381.

18. McLigeyo SO. The pattern of geriatric admissions in the medical wards at the Kenyatta National Hospital. East Afr Med J. 1993;70:37-39.

19. Redondo-Sendino A, Guallar-Castillón P, Banegas JR, Rodríguez-Artalejo F. Gender differences in the utilization of health-care services among the older adult population of Spain. BMC Public Health. 2006;6:155.

How to cite this article: Sankar D R, Kumar D. S. S. A study of medical problems and their outcome in the elderly patients attending the geriatric out patient department of a tertiary care teaching hospital in south India. J Community Health Manag. 2018;5(4):197-201. 